\title{
PURE RED CELL APLASIA INDUCED BY ERYTHROPOIETIN
}

\author{
Mihajlović Filip, Milosavljević Aleksandar, Đurić Dušan ${ }^{1}$ \\ ${ }^{1}$ Faculty of medical sciences, University of Kragujevac, Serbia
}

Primljen / Received 14. 12. 2017. god.

Abstract: Introduction: Recombined human erythropoietin has been present in clinical practice for more than 20 years, in these therapeutic indications: anemia in kidney insufficiency, anemia during chemotherapy of tumors, prevention of anemias that appear in premature born babies, it is used for increasing autologous blood cell levels before blood transfusion, AIDS joined anemia (intensified by zidovudine), anemia joined with chronic inflammatory conditions such as rheumatoid arthritis(still in research phase). During the course of Erythropoietin treatment side effects have been noticed, that include multiple organ systems, and have different levels of frequency. Major number of studies shows connection between erythropoietin treatment and bone marrow aplasia, but small number of them states clearly defined side-effect that explains this phenomenon.

Objective: Goal of this paper is to analyze available case studies of bone marrow aplasia during erythropoietin application, access their quality and causality of every study.

Method: Research of literature used for the preparation of this systematic review has been conducted during the period of November- December 2017. In search for literature medical base PubMed has been used. Inclusion criteria were: available full article, publications in English language, publications conducted on humans, and case report studies. Eight studies passed selection.

Results: results were presented by 5 charts: documentation size, credibility, number of case study reports of side-effects in the paper that was graded, Naranjo causality score, data extraction chart. Mean grade value of the studies quality was 7,0 , while mean Naranjo score was 6,6 .

Key words: erythropoietin, erythropoietin side effects, anti-erythropoietin antibodies, pure red cell aplasia.

\section{INTRODUCTION}

Erythropoietin regulates the development of erythroid cell line from proerythroblast to erythrocyte. Loss of blood, and/or low tissue oxygen levels are the
Prihvaćen/Accepted 10. 03. 2018. god.

basic stimulus for its release into the bloodstream. Erythropoietin is produced in the juxtaglomerular cells of the kidney and for one small part in the macrophages. Basic effect of erythropoietin is to stimulate proliferation of erythrocyte precursor cells and create new erythrocytes. As a consequence of kidney disease, due to diminished erythropoietin synthesis, anemia can occur (1).

Chronic kidney disease (CKD) encompasses a spectrum of different pathophysiologic processes, caused by different etiologic factors, associated with abnormal kidney function and a progressive decline in glomerular filtration rate, often leading to the end stage renal disease (ESRD). Term end-stage renal disease represents a stage of CKD where the accumulation of toxins, fluid, and electrolytes normally excreted by the kidneys results in the uremic syndrome. This syndrome leads to death unless the toxins are removed by renal replacement therapy, using dialysis or kidney transplantation (2). Anemia occurs in more than $80 \%$ of patients with compromised kidney function (3). In patients with CKD the main cause of normocytic, normochromic anemia is insufficient erythropoietin production in damaged kidneys (4). Factors contributing to the occurrence of anemia can be: loss of iron due to various diagnostic procedures, blood retention in the dialysis machine, gastrointestinal bleeding, hyperparathyroidism, acute and chronic inflammatory diseases, aluminum toxicity, lack of folic-acid (5).

In order to solve the problem of anemia in dialysis patients, a group of researchers in 1985 cloned a human gene for erythropoietin. Two years later, the successful results of this study were published by The New England Journal of Medicine, latter In 1989, FDA approved the use of this hormone, in patients with CKD on dialysis (6). Erythropoietin is a glycoprotein structured hormone. The average molecular weight is 32, 00040,000 Daltons. The protein portion accounts for about $58 \%$ of the molecular weight (165 amino acids). Within its molecule there are four carbohydrate 
chains linked through $3 \mathrm{~N}$-glycosidic linkages and one $\mathrm{O}$-glycoside linkage. The amino acid sequence and carbohydrate part in recombinant erythropoietin are identical to those in the endogenous human erythropoietin isolated from the urine of anemic mice (7). Recombinant human erythropoietin has been present in clinical practice for more than 20 years, for therapeutic indications: anemia in renal failure, anemia during chemotherapy in malignant tumors, prevention of anemia occurring in preterm infants, an increase in autologous blood production before donation, anemia in AIDS, (potentiated by zidovudine), anemia in chronic inflammatory conditions such as rheumatoid arthritis (still in research phase) (8).

Adverse effects of erythropoietin include: flu-like syndrome that is transient, hypertension, which can cause encephalopathy with headache disorientation and sometimes convulsions. Iron deficit can occur due to increased need for it caused by intense erythropoiesis. Increase in blood viscosity and hematocrit can also occur, which can lead to thrombosis. The emphasis in writing this paper will be the red cell aplasia due to the development of anti-erythropoietic antibodies during treatment with any erythropoietin medication (9). The period between 1998 and 2001 was marked by an increased concern for safety of erythropoietin use, due to the increased number of registered cases of pure red cell aplasia caused by neutralizing, anti-erythropoietin antibodies. A severe anemia, resistant to erythropoietin therapy, has developed in this group of patients. Pure red cell aplasia (PRCA) was registered primarily in those patients who were treated with subcutaneous administration of erythropoietin alpha, and it is attributed to changes in the product stabilization formulation and failure to comply with the cold chain principle (7). Isolated red cell aplasia due to the use of erythropoietin is a bone marrow disease followed by severe anemia due to hypoplasia of erythroid lineage precursor cells. The other precursors are not affected by this disease, so the patients present with normochromic, normocytic anemia with values of thrombocytes and neutrocytes within the normal range (9).

The aim of this paper is to analyze the available case reports of red cell aplasia due to erythropoietin use, evaluation of their quality and causality for each study separately.

\section{MATERIAL AND METHOD}

For the purposes of this systematic review article a search through PubMed database was performed. Before the search, the following criteria were set: in relation to the type of article - case report, in terms of availability - entire texts and in terms of population-humans were population in the searched studies. The search was carried out in November 2017 and the inclusion criteria were: Free full article, English language, Case report studies, Studies that describe the occurrence of bone marrow disorders due to erythropoietin use

The exclusion criteria were: studies conducted on animals, studies that are not "case reports" designs, "Letters to editor", case reports available in the form of an abstract.

Only studies conducted on humans were taken into consideration, and there were no restrictions in terms of age and sex. Keyword searched: erythropoietin hematological side effects, resulting in 15 studies; anti-erythropoietin antibodies in the search result was 30 studies; erythropoietin inducted pure red cell aplasia, resulting in 18 studies. Several studies appeared as a result of the search after using all three keyword combinations, and the last used combination of keywords included all the studies that appeared as search results. The most important criteria for inclusion were related to the following aspects: the study had to have one to two case reports; these reports were related to bone marrow aplasia due to erythropoietin administration. Diagnostic data for the bone marrow aplasia was necessary, since this side effect of erythropoietin is the subject of research in this paper. This paper includes 8 different studies.

Studies that did not pass the selection deviated from the required design of the study and the desired intervention. Studies that showed series of cases were excluded. Also, studies in which, instead of the administration of erythropoietin, patients received another drug or bone marrow aplasia occurred as a result of another disease, were excluded.

\section{RESULTS}

The results of this review paper are shown in the table's bellow, for all the studies that met the inclusion criteria. The assessment of the volume of the documentation for each study equals the sum of the elements present; to indicate the presence, not the absence of an element. The aim is to determine whether the data in the study are complete in order to draw conclusions about the cause-and-effect relationship between drug use and the occurrence of an adverse event.

The assessment of the credibility of each study is equal as the sum of the elements present; to indicate the presence, not the absence of an element. The goal of the table is to display information about when and where the event occurred and who recorded it.

By looking at Table $2 \ldots$ We note that all selected studies contain information on the time and location of the adverse event, as well as information about a medi- 
Table 1. Volume of the documentation

\begin{tabular}{|c|c|c|c|c|c|c|c|c|}
\hline studies & $\begin{array}{l}\text { Has the time } \\
\text { interval from } \\
\text { application of } \\
\text { the medication } \\
\text { to appearance } \\
\text { of adverse } \\
\text { effect been } \\
\text { noted? }\end{array}$ & $\begin{array}{c}\text { Has } \\
\text { dechallenge } \\
\text { been described? }\end{array}$ & $\begin{array}{l}\text { Has rechallenge } \\
\text { been described? }\end{array}$ & $\begin{array}{l}\text { Has that } \\
\text { adverse effect } \\
\text { already been } \\
\text { documented? }\end{array}$ & $\begin{array}{c}\text { Have there } \\
\text { been alternative } \\
\text { causes of side } \\
\text { effect? }\end{array}$ & $\begin{array}{l}\text { Has there been } \\
\text { diagnostic } \\
\text { confirmation? }\end{array}$ & $\begin{array}{l}\text { Is there rational } \\
\text { explanation for } \\
\text { the mechanism } \\
\text { of adverse } \\
\text { effect? }\end{array}$ & points \\
\hline $\begin{array}{l}\text { study } \\
1(10)\end{array}$ & yes & yes & no & yes & no & yes & yes & 3 \\
\hline $\begin{array}{l}\text { study } \\
2(11)\end{array}$ & yes & yes & no & yes & no & yes & yes & 3 \\
\hline $\begin{array}{l}\text { study } \\
3(12)\end{array}$ & yes & yes & no & yes & no & yes & yes & 3 \\
\hline $\begin{array}{l}\text { study } \\
4(13)\end{array}$ & yes & yes & no & yes & no & yes & yes & 3 \\
\hline $\begin{array}{l}\text { study } \\
5(14)\end{array}$ & yes & yes & no & yes & no & yes & yes & 3 \\
\hline $\begin{array}{l}\text { study } \\
6(15)\end{array}$ & yes & yes & no & yes & no & yes & yes & 3 \\
\hline $\begin{array}{l}\text { study } \\
7(16)\end{array}$ & yes & yes & no & yes & no & yes & yes & 3 \\
\hline $\begin{array}{l}\text { study } \\
8(17)\end{array}$ & yes & yes & no & yes & no & yes & yes & 3 \\
\hline
\end{tabular}

scoring system: $>4$ elements -3 points; $2-4$ elements -2 points; 1 element -1 point; 0 elements - 0 points

Table 2. Credibility of the studies

\begin{tabular}{|c|c|c|c|}
\hline study & Are there timeline data? & $\begin{array}{c}\text { Are there data of location } \\
\text { of the adverse effect? }\end{array}$ & $\begin{array}{c}\text { Are there data about } \\
\text { medical worker that made } \\
\text { the report? }\end{array}$ \\
\hline study 1 & yes & yes & yes \\
\hline study 2 & yes & yes & yes \\
\hline study 3 & yes & yes & yes \\
\hline study 4 & yes & yes & yes \\
\hline study 5 & yes & yes & yes \\
\hline study 6 & yes & yes & yes \\
\hline study 7 & yes & grade & 3 \\
\hline study 8 & yes & & \\
\hline number of elements present & & \\
\hline 3 & & & \\
\hline
\end{tabular}

cal worker who has documented the undesirable effects. The presence of these data points to the significant credibility of the selected studies. The number of cases described in the analyzed studies: one case carries a grade of 1, while studies describing 2 or 3 cases carry a score of 2 . 
Table 3. Number of cases descr

\begin{tabular}{|c|c|}
\hline study & number of cases described \\
\hline study 1 & 1 \\
\hline study 2 & 1 \\
\hline study 3 & 1 \\
\hline study 4 & 1 \\
\hline study 5 & 1 \\
\hline study 6 & 1 \\
\hline study 7 & 1 \\
\hline study 8 & 1 \\
\hline
\end{tabular}

Table 4. The number of elements present of cases

\begin{tabular}{|c|c|}
\hline number of elements present & grade \\
\hline 1 & 1 \\
\hline$>1$ & 2 \\
\hline
\end{tabular}

Naranjo score was used to evaluate the cause-and-effect relationship between drug use and the occurrence of an adverse event. It contains questions relating to the time period between the use of the medicine and the occurrence of an adverse reaction, what happens after the cessation of use, or the re-administration of the drug, whether there are alternative causes for the occurrence of an adverse event, whether there is a laboratory confirmation, etc.

Table 5. Naranjo causality score

\begin{tabular}{|l|c|c|c|}
\hline \multicolumn{1}{|c|}{ Naranjo causality score } & & \\
\hline \multicolumn{1}{|c|}{ question } & yes & no & $\begin{array}{c}\text { do not } \\
\text { know }\end{array}$ \\
\hline 1. Are there previous conclusive reports on this reaction? & +1 & 0 & 0 \\
\hline 2. Did the adverse events appear after the suspected drug was given? & +2 & -1 & 0 \\
\hline 3. Did the adverse reaction improve when the drug was discontinued or a specific antagonist was given? & +1 & 0 & 0 \\
\hline 4. Did the adverse reaction appear when the drug was readministered? & +2 & -1 & 0 \\
\hline 5. Are there alternative causes that could have caused the reaction? & -1 & +2 & 0 \\
\hline 6. Did the reaction reappear when a placebo was given? & -1 & +1 & 0 \\
\hline 7. Was the drug detected in any body fluid in toxic concentrations? & +1 & 0 & 0 \\
\hline 8. Was the reaction more severe when the dose was increased, or less severe when the dose was decreased? & +1 & 0 & 0 \\
\hline 9. Did the patient have a similar reaction to the same or similar drugs in any previous exposure? & +1 & 0 & 0 \\
\hline 10. Was the adverse event confirmed by any objective evidence? & +1 & & 0 \\
\hline $\begin{array}{l}\text { Scoring: } \\
\geq 9=\text { definite ADR, 5-8 = probable ADR, 1-4 = possible ADR, } 0=\text { doubtful ADR }\end{array}$ & & & \\
\hline
\end{tabular}

\begin{tabular}{|c|c|c|c|c|c|c|c|c|c|c|c|}
\hline study & $\begin{array}{l}\text { Are there } \\
\text { previous } \\
\text { conclusive } \\
\text { reports on } \\
\text { this } \\
\text { reaction? }\end{array}$ & $\begin{array}{l}\text { Did the } \\
\text { adverse } \\
\text { events } \\
\text { appear } \\
\text { after the } \\
\text { suspected } \\
\text { drug was } \\
\text { given? }\end{array}$ & $\begin{array}{l}\text { Did the } \\
\text { adverse } \\
\text { reaction } \\
\text { improve } \\
\text { when the } \\
\text { drug was } \\
\text { discontinu } \\
\text { ed or a } \\
\text { specific } \\
\text { antagonist } \\
\text { was given? }\end{array}$ & $\begin{array}{l}\text { Did the } \\
\text { adverse } \\
\text { reaction } \\
\text { appear } \\
\text { when the } \\
\text { drug was } \\
\text { readminist } \\
\text { ered? }\end{array}$ & $\begin{array}{l}\text { Are there } \\
\text { alternative } \\
\text { causes that } \\
\text { could have } \\
\text { caused the } \\
\text { reaction? }\end{array}$ & $\begin{array}{l}\text { Did the } \\
\text { reaction } \\
\text { reappear } \\
\text { when a } \\
\text { placebo } \\
\text { was given? }\end{array}$ & $\begin{array}{l}\text { Was the } \\
\text { drug } \\
\text { detected in } \\
\text { any body } \\
\text { fluid in } \\
\text { toxic } \\
\text { concentrati } \\
\text { ons? }\end{array}$ & $\begin{array}{c}\text { Was the } \\
\text { reaction } \\
\text { more } \\
\text { severe } \\
\text { when the } \\
\text { dose was } \\
\text { increased, } \\
\text { or less } \\
\text { severe } \\
\text { when the } \\
\text { dose was } \\
\text { decreased? }\end{array}$ & $\begin{array}{c}\text { Did the } \\
\text { patient } \\
\text { have a } \\
\text { similar } \\
\text { reaction to } \\
\text { the same } \\
\text { or similar } \\
\text { drugs in } \\
\text { any } \\
\text { previous } \\
\text { exposure?? }\end{array}$ & $\begin{array}{c}\text { Was the } \\
\text { adverse } \\
\text { event } \\
\text { confirmed } \\
\text { by any } \\
\text { objective } \\
\text { evidence? }\end{array}$ & grade \\
\hline $1_{(10)}$ & $\begin{array}{c}+1 \\
\text { (yes) }\end{array}$ & $\begin{array}{c}+2 \\
\text { (yes) }\end{array}$ & $\begin{array}{c}+1 \\
\text { (yes) }\end{array}$ & $\begin{array}{l}0 \text { (don't } \\
\text { know) }\end{array}$ & $\begin{array}{l}+2 \\
\text { (no) }\end{array}$ & $\begin{array}{l}0 \text { (don’t } \\
\text { know) }\end{array}$ & $\begin{array}{l}0 \text { (don’t } \\
\text { know) }\end{array}$ & $\begin{array}{l}0 \text { (don’t } \\
\text { know) }\end{array}$ & $\begin{array}{l}0 \text { (don't } \\
\text { know) }\end{array}$ & $\begin{array}{c}+1 \\
\text { (yes) }\end{array}$ & $\begin{array}{c}7 \\
\text { (probable) }\end{array}$ \\
\hline $2(11)$ & $\begin{array}{c}+1 \\
\text { (yes) }\end{array}$ & $\begin{array}{c}+2 \\
\text { (yes) }\end{array}$ & $\begin{array}{c}+1 \\
\text { (yes) }\end{array}$ & $\begin{array}{l}0 \text { (don’t } \\
\text { know) }\end{array}$ & $\begin{array}{l}+2 \\
\text { (no) }\end{array}$ & $\begin{array}{l}0 \text { (don’t } \\
\text { know) }\end{array}$ & $\begin{array}{l}0 \text { (don’t } \\
\text { know) }\end{array}$ & $\begin{array}{l}0 \text { (don’t } \\
\text { know) }\end{array}$ & $\begin{array}{l}0 \text { (don’t } \\
\text { know) }\end{array}$ & $\begin{array}{c}+1 \\
\text { (yes) }\end{array}$ & $\begin{array}{c}7 \\
\text { (probable) }\end{array}$ \\
\hline $3(12)$ & $\begin{array}{c}+1 \\
\text { (yes) }\end{array}$ & $\begin{array}{c}+2 \\
\text { (yes) }\end{array}$ & $\begin{array}{l}0 \text { (don’t } \\
\text { know) }\end{array}$ & $\begin{array}{l}0 \text { (don’t } \\
\text { know) }\end{array}$ & $\begin{array}{l}+2 \\
\text { (no) }\end{array}$ & $\begin{array}{l}0 \text { (don’t } \\
\text { know) }\end{array}$ & $\begin{array}{l}0 \text { (don’t } \\
\text { know) }\end{array}$ & $\begin{array}{l}0 \text { (don’t } \\
\text { know) }\end{array}$ & $\begin{array}{l}0 \text { (don’t } \\
\text { know) }\end{array}$ & $\begin{array}{c}+1 \\
\text { (yes) }\end{array}$ & $\begin{array}{c}6 \\
\text { (probable) }\end{array}$ \\
\hline $4_{(13)}$ & $\begin{array}{c}+1 \\
\text { (yes) }\end{array}$ & $\begin{array}{l}+2 \\
\text { (yes) }\end{array}$ & $\begin{array}{c}+1 \\
(\mathrm{yes})\end{array}$ & $\begin{array}{l}0 \text { (don’t } \\
\text { know) }\end{array}$ & $\begin{array}{l}+2 \\
\text { (no) }\end{array}$ & $\begin{array}{l}0 \text { (don't } \\
\text { know) }\end{array}$ & $\begin{array}{l}0 \text { (don’t } \\
\text { know) }\end{array}$ & $\begin{array}{l}0 \text { (don’t } \\
\text { know) }\end{array}$ & $\begin{array}{l}0 \text { (don’t } \\
\text { know) }\end{array}$ & $\begin{array}{l}+1 \\
\text { (yes) }\end{array}$ & $\begin{array}{c}7 \\
\text { (probable) }\end{array}$ \\
\hline $5_{(14)}$ & $\begin{array}{c}+1 \\
\text { (yes) }\end{array}$ & $\begin{array}{c}+2 \\
\text { (yes) }\end{array}$ & $\begin{array}{c}+1 \\
\text { (yes) }\end{array}$ & $\begin{array}{l}0 \text { (don’t } \\
\text { know) }\end{array}$ & $\begin{array}{l}+2 \\
\text { (no) }\end{array}$ & $\begin{array}{l}0 \text { (don’t } \\
\text { know) }\end{array}$ & $\begin{array}{l}0 \text { (don’t } \\
\text { know) }\end{array}$ & $\begin{array}{l}0 \text { (don’t } \\
\text { know) }\end{array}$ & $\begin{array}{l}0 \text { (don’t } \\
\text { know) }\end{array}$ & $\begin{array}{c}+1 \\
\text { (yes) }\end{array}$ & $\begin{array}{c}7 \\
\text { (probable) }\end{array}$ \\
\hline $6(15)$ & $\begin{array}{c}+1 \\
\text { (yes) }\end{array}$ & $\begin{array}{c}+2 \\
\text { (yes) }\end{array}$ & $\begin{array}{c}+1 \\
\text { (yes) }\end{array}$ & $\begin{array}{l}0 \text { (don’t } \\
\text { know) }\end{array}$ & $\begin{array}{l}+2 \\
\text { (no) }\end{array}$ & $\begin{array}{l}0 \text { (don’t } \\
\text { know) }\end{array}$ & $\begin{array}{l}0 \text { (don’t } \\
\text { know) }\end{array}$ & $\begin{array}{l}0 \text { (don't } \\
\text { know) }\end{array}$ & $\begin{array}{l}0 \text { (don’t } \\
\text { know) }\end{array}$ & $\begin{array}{c}+1 \\
\text { (yes) }\end{array}$ & $\begin{array}{c}7 \\
\text { (probable) }\end{array}$ \\
\hline $7_{(16)}$ & $\begin{array}{c}+1 \\
\text { (yes) }\end{array}$ & $\begin{array}{l}+2 \\
\text { (yes) }\end{array}$ & $\begin{array}{l}0 \text { (don't } \\
\text { know) }\end{array}$ & $\begin{array}{l}0 \text { (don’t } \\
\text { know) }\end{array}$ & $\begin{array}{l}+2 \\
\text { (no) }\end{array}$ & $\begin{array}{l}0 \text { (don’t } \\
\text { know) }\end{array}$ & $\begin{array}{l}0 \text { (don’t } \\
\text { know) }\end{array}$ & $\begin{array}{l}0 \text { (don't } \\
\text { know) }\end{array}$ & $\begin{array}{l}0 \text { (don’t } \\
\text { know) }\end{array}$ & $\begin{array}{c}+1 \\
\text { (yes) }\end{array}$ & $\begin{array}{c}6 \\
\text { (probable) }\end{array}$ \\
\hline $8(17)$ & $\begin{array}{c}+1 \\
\text { (yes) }\end{array}$ & $\begin{array}{c}+2 \\
\text { (yes) }\end{array}$ & $\begin{array}{l}0 \text { (don't } \\
\text { know) }\end{array}$ & $\begin{array}{l}0 \text { (don’t } \\
\text { know) }\end{array}$ & $\begin{array}{l}+2 \\
\text { (no) }\end{array}$ & $\begin{array}{l}0 \text { (don’t } \\
\text { know) }\end{array}$ & $\begin{array}{l}0 \text { (don’t } \\
\text { know) }\end{array}$ & $\begin{array}{l}0 \text { (don’t } \\
\text { know) }\end{array}$ & $\begin{array}{l}0 \text { (don’t } \\
\text { know) }\end{array}$ & $\begin{array}{c}+1 \\
\text { (yes) }\end{array}$ & $\begin{array}{c}6 \\
\text { (probable) }\end{array}$ \\
\hline
\end{tabular}


Data extraction: The goal of the table is to syn- ent's sex and age, the dose of erythropoietin, how long thesize data on each case analyzed, such as the pati- was the patient monitored and the outcome.

Table 6. Data extraction

\begin{tabular}{|c|c|c|c|c|c|c|c|}
\hline $\begin{array}{l}\text { extraction } \\
\text { date }\end{array}$ & study name and authors & $\begin{array}{l}\text { study } \\
\text { quality } \\
\text { grade }\end{array}$ & $\begin{array}{c}\text { study } \\
\text { population }\end{array}$ & intervention & outcome & $\begin{array}{l}\text { length of } \\
\text { monitoring }\end{array}$ & $\begin{array}{c}\text { Naranjo } \\
\text { score }\end{array}$ \\
\hline 30. 11. 2017. & $\begin{array}{c}\text { Yoshimi M, Kadowaki } \\
\text { Y, Kikuchi } \\
\text { Y, Takahashi T. Coomb s negative } \\
\text { autoimmune hemolytic anemia followed } \\
\text { by anti-erythropoetin receptor } \\
\text { antibodyassociated pure red cell aplasia: } \\
\text { A case report and review of literature. }{ }^{[10]}\end{array}$ & 7 & $\begin{array}{c}\text { female, } \\
26 \text { years old }\end{array}$ & $\begin{array}{c}\text { erythropoietin } \\
\text { 1000IU/ } \\
\text { weeksubcutaneous }\end{array}$ & $\begin{array}{l}\text { withdrawal of } \\
\text { adverse effect and } \\
\text { bone marrow } \\
\text { reactivation }\end{array}$ & 4 weeks & 7 \\
\hline 30. 11. 2017. & $\begin{array}{l}\text { Lucio Manenti, Augusto Vaglio. } \\
\text { Pure red cell aplasia followed by } \\
\text { disseminated intravascular coagulation in } \\
\text { a haemodialysis patient receiving } \\
\text { erythropoietin- }-\beta{ }^{[11]}\end{array}$ & 7 & $\begin{array}{c}\text { male, } \\
79 \text { years old }\end{array}$ & $\begin{array}{l}\text { erythropoietin beta, } \\
\text { 4000IU/week } \\
\text { subcutaneous }\end{array}$ & $\begin{array}{l}\text { withdrawal of } \\
\text { adverse effect and } \\
\text { bone marrow } \\
\text { reactivation }\end{array}$ & 9 weeks & 7 \\
\hline 30. 11. 2017. & $\begin{array}{l}\text { Okoshi Y et al. } \\
\text { A patient with acquired pure red cell } \\
\text { aplasia showing a positive antiglobulin } \\
\text { test and the presence of inhibitor against } \\
\text { erythroid precursors. }{ }^{[12]}\end{array}$ & 7 & $\begin{array}{c}\text { male, } \\
66 \text { years old }\end{array}$ & $\begin{array}{l}\text { erythropoietin beta, } \\
10000 \mathrm{IU} / \text { week } \\
\text { subcutaneous }\end{array}$ & $\begin{array}{l}\text { withdrawal of } \\
\text { adverse effect }\end{array}$ & 8 weeks & 6 \\
\hline 30. 11. 2017. & $\begin{array}{c}\text { Renaud Snanoudjaet al. Recovery from } \\
\text { Pure Red Cell Aplasia } \\
\text { Caused by Anti-Erythropoietin } \\
\text { Antibodies After Kidney } \\
\text { Transplantation. }{ }^{[13]}\end{array}$ & 7 & $\begin{array}{l}\text { patient sex } \\
\text { and age not } \\
\text { available }\end{array}$ & $\begin{array}{l}\text { erythropoietin } \\
\text { alpha, } \\
1000 \text { IU then } \\
4000 \mathrm{IU} / \text { week } \\
\text { subcutaneous }\end{array}$ & $\begin{array}{l}\text { withdrawal of } \\
\text { adverse effect and } \\
\text { bone marrow } \\
\text { reactivation }\end{array}$ & 6 weeks & 7 \\
\hline 30. 11. 2017. & $\begin{array}{l}\text { Lee } \mathrm{H} \text {, Yang J, Kim H et al. } \\
\text { Improvement in erythropoieisstimulating } \\
\text { agentinduced pure red-cell aplasia by } \\
\text { introduction of darbepoetin- } \alpha \text { when the } \\
\text { anti-erythropoietin antibody titer declines } \\
\text { spontaneously. }\end{array}$ & 7 & $\begin{array}{c}\text { female, } \\
36 \text { years old }\end{array}$ & $\begin{array}{l}\text { erythropoietin } \\
\text { alpha } \\
3000-6000 \\
\text { IU/week } \\
\text { subcutaneous }\end{array}$ & $\begin{array}{l}\text { withdrawal of } \\
\text { adverse effect and } \\
\text { bone marrow } \\
\text { reactivation }\end{array}$ & 5 weeks & 7 \\
\hline 30. 11.2017. & $\begin{array}{c}\text { Ashwin Asari, } \\
\text { Ram Gokal. Pure Red Cell Aplasia } \\
\text { Secondary to Epoetin alpha Responding } \\
\text { to Darbepoetin Alpha in a Patient on } \\
\text { Peritoneal Dialysis. }{ }^{[15]}\end{array}$ & 7 & $\begin{array}{c}\text { male, } \\
82 \text { years old }\end{array}$ & $\begin{array}{l}\text { erythropoietin beta } \\
\text { 3000IU/week } \\
\text { intraperitoneal }\end{array}$ & $\begin{array}{l}\text { withdrawal of } \\
\text { adverse effect }\end{array}$ & 8 weeks & 7 \\
\hline 30. 11. 2017. & $\begin{array}{l}\text { Gertrud Weber, Johann Gross, } \\
\text { Arno Kromminga, Hans-H. Loew, } \\
\text { Kai-Uwe Eckardt. } \\
\text { Allergic Skin and Systemic Reactions in } \\
\text { a Patient with Pure Red Cell Aplasia and } \\
\text { Anti-Erythropoietin Antibodies } \\
\text { Challenged with Different } \\
\text { Epoetins. }\end{array}$ & 7 & $\begin{array}{c}\text { female, } \\
48 \text { years old }\end{array}$ & $\begin{array}{l}\text { erythropoietin } \\
\text { alpha } \\
3 \times 2000 I U / \text { week } \\
\text { subcutaneous }\end{array}$ & $\begin{array}{l}\text { withdrawal of } \\
\text { adverse effect and } \\
\text { bone marrow } \\
\text { reactivation }\end{array}$ & 7 weeks & 6 \\
\hline 30. 11. 2017. & $\begin{array}{l}\text { Chng WJ, Tan LK, Liu TC. Cyclosporine } \\
\text { treatment for patients with CRF who } \\
\text { developed pure red blood cell aplasia } \\
\text { following EPO therapy. }{ }^{[17]}\end{array}$ & 7 & $\begin{array}{l}\text { patient sex } \\
\text { and age not } \\
\text { available }\end{array}$ & $\begin{array}{l}\text { erythropoietin } \\
\text { alpha } \\
\text { 2000/ week } \\
\text { subcutaneous }\end{array}$ & $\begin{array}{l}\text { withdrawal of } \\
\text { adverse effect }\end{array}$ & 7 weeks & 6 \\
\hline
\end{tabular}




\section{DISCUSSION}

The primary aim of this systematic review article was to conduct a detailed search of medical databases, selecting appropriate studies, and processing, and pooling the results of these studies, to answer the question of whether there is a connection between the use of erythropoietin and bone marrow aplasia. In addition, this paper has the task of examining the literature on the safety of the use of erythropoietin, assessing the seriousness of the undesirable effect, and the mechanism of their emergence. We can see that in all the selected studies, the time interval from the use of the drug to the occurrence of an adverse drug reaction is described. All studies also contain information on what happens after the cessation of the drug (Dechallenge), while (Rechallenge) has not been performed in any study. This points to the fact that doctors mostly avoid exposure of patients to drugs that are suspected of having caused an unwanted effect by prior use.

The average value of the evaluation of the quality of the study was 7.0. The results of this systematic review article in terms of causality are similar to the results of the previous studies on the same subject. The calculated mean value of Naranjo causality score is 6.6 (5-8 probable causality), we can conclude that there is a likely correlation between the use of erythropoietin and bone marrow aplasia. The average age of patients was 56.1 years (the oldest patient was 82 years old and the youngest 26 years old). Three patients were female, three male, while for two patients, data on age and sex were not given. Erythropoietin-induced bone marrow aplasia, as a serious (life-threatening) adverse effect, carries the risk of aplastic anemia and aggravation of disseminated intravascular coagulation, as described in the analyzed studies. Particularly sensitive patients are those who start erythropoietin therapy after receiving kidney transplant, in order to prevent anemia (15, 16). The most used erythropoietin forms were erythropoietin alpha and beta (17). Erythropoietin was mostly used for described therapeutic indication which is anemia in patients on hemodialysis, one of the studies included patient on peritoneal dialysis which received erythropoietin (17).

Generally, the aplasia appeared several weeks ( 8 to 10 on average) after erythropoietin administration, which is explained by the lifetime of the erythrocytes synthesized prior to administration, which is about 120 days. After their degradation in the spleen, the bone marrow damaged by the use of erythropoietin would no longer produce new erythrocytes, and signs of anemia would appear (13). The first symptoms that caused these patients to visit their nephrologist or the general physician were dizziness, fatigue, decreased appetite and rapid heart rate. In suspected cases, routine blood tests in which decreased erythrocytes were observed (below $4-4.5^{*} 10^{12} / 1$ for women and $4.5-5 * 10^{12} / 1$ for men), decreased hemoglobin levels (below $120 \mathrm{~g} / \mathrm{l}$ ) and hematocrit (below 0.45\%) (17). The literature states that there is a mechanism of action that causes erythropoietin to cause bone marrow aplasia.

Bone marrow aplasia, with a previously history of erythropoietin administration, must be confirmed by laboratory tests. These include measuring hemoglobin levels, hematocrit number of erythrocytes, and serum iron levels $(13,14,15)$. Therefore, each of the analyzed studies contained a mandatory laboratory confirmation for this adverse effect. In addition to these routine laboratory analyzes, for the final confirmation of erythropoietin-induced aplasia, staining of bone marrow samples was performed. Immunochemical methods and staining of bone marrow preparations, provided an explanation of the mechanism by which erythropoietin leads to bone marrow aplasia. This led to the detection of neutralizing anti-erythropoietin antibodies responsible for the occurrence of this adverse effect $(13,16,17$.) In order for the bone marrow to recover, it is necessary to exclude erythropoietin and administer immunosuppressant (cyclosporine) (17). To monitor the rate of recovery, an analysis of the number of reticulocytes was carried out which proved that after a certain period of time the bone marrow starts regenerating, producing the peripheral blood young erythrocytes-reticulocytes (13).

Considering that men physiologically have a higher number of erythrocytes due to hormonal status, bone marrow is regenerated faster in a male patients, as well as in younger patients compared to the older ones (17). As reported in the available literature, erythropoietin causes bone marrow aplasia rarely. In all cases after cessation of the drug that was suspected to lead to aplasia of the red cell line, the undesirable effect has withdrawn (10-17.) In all eight studies, there are no alternative causes that could trigger the investigated adverse event, which is of great importance for linking the investigated drug with an unwanted event. All of the above facts speak of the likely causality between the use of erythropoietin and the onset of bone marrow aplasia.

\section{CONCLUSION}

Treatment of anemia in dialysis patients with erythropoietin can, rarely leads to serious (life-threatening) adverse effects. Symptoms and signs of bone marrow aplasia can be recognized and prevented early by careful monitoring of the patients by the nephrologist or clinical pharmacologist. There is clear risk for occurrence of this adverse effect, so careful use and do- 
sing of erythropoietin are to be advised. It is also important to inform patients about the first symptoms of aplasia, but also to increase the involvement of healthcare professionals in terms of reporting this adverse effect to the National Pharmacovigilance Center.

\section{Declaration of interest}

There is no conflict of interest between the authors of this paper.

\section{Acknowledgement}

I thank my colleague and coauthor, future doctor of medicine Milosavljevic Aleksandar, for all help and support he has given me during the writing of this paper.
Abbreviations
AIDS - acquired immunodeficiency syndrome
CKD - chronic kidney disease
ESRD - end stage renal disease
FDA - Food and Drug Administration
ADR - Adverse drug reaction

\section{Licensing}

This work is licensed under a Creative Commons Attribution 4.0 International (CC BY 4.0) Licence

\title{
Sažetak
}

\section{APLAZIJA KOŠTANE SRŽI USLED PRIMENE ERITROPOETINA}

\author{
Mihajlović Filip, Milosavljević Aleksandar, Đurić Dušan ${ }^{1}$ \\ ${ }^{1}$ Fakultet medicinskih nauka Kragujevac, Univerzitet u Kragujevcu
}

Uvod: Rekombinovani humani eritropoetin prisutan je u kliničkoj praksivišeod 20 godina, za terapijske indikacije: anemija kod bubrežne insuficijencije, anemija u toku hemioterapije kod malignih tumora, prevencija anemija koje se javljaju kod nedonoščadi, za povećanje proizvodnje autologne krvi pre donacije, anemija kod AIDS-a (potencirana primenom zidovudina), anemija u hroničnim zapaljenskim stanjima kao što je reumatoidni artritis (u fazi istraživanja). Tokom terapije eritropoetinom, uočena su neželjena dejstva na nivou različitih sistema organa, i različitog stepena učestalosti. Veliki broj studija ukazuje na povezanost između primene eritropetina i pojave aplazije koštane srži, ali mali broj njih navodi jasno definisano neželjeno dejstvo koje ovu povezanost objašnjava.

Cilj rada: Cilj ovog rada je analiza dostupnih prikaza slučajeva aplazije koštane srži usled primene eritropoetina, procena njihovog kvaliteta i kauzalnosti za svaku studiju pojedinačno.

\section{REFERENCES}

1. Ristić Z. Hematopoiesis. In: Ristić M, Ristić S. Hematology with transfusiology. Beograd 2011.p.54.

2. Skorecki K Chronic kidney failure. In: Harrisons principles of internal medicine 15th edition, Beograd. Brad - Fin \& Romanov, 2004. p.1511.

3. Lewin A. Indetification of patients and risk factors in chronic kidney disease-ebaluating risk factors and therapeutic strategies. Nephro 1Dail Transplant. 2001; 16 (Supp1 7): 57- 60.

4. Barbone FP, Johnson DL Farrell FX, Collins A, Middleton SA, McMahon FJ, et al. New epoetin molecules and novel therapeutic approaches.Nephrol Dial Transplant. 1999; 14 (Suppl 2): 80-4.
Metod: Pretraga literature koja je korišćena za izradu ovog sistematskog preglednog članka izvršenaje u periodu novembar - decembar 2017. godine. Za pretraživanje literature korišćena je medicinska baza podataka. PubMed. Kriterijumi za uključenje bili su: publikacije dostupne u celosti, publikacije na engleskom jeziku, publikacije sprovedene na ljudima, i studije $u$ kojima je opisan klinički slučaj (case report). Osam studija je prošlo selekciju.

Rezultati: Rezultati su tabelarno predstavljeni kroz pet tabela: opsežnos tdokumentacije, verodostojnost, broj opisanih prikaza slučajeva neželjenih dejstava u radu koji se ocenjuje, Naranjo skor kauzalnosti, tabela za ekstrakciju podataka. Srednja vrednost ocena kvaliteta studija iznosila je 7,0 dok je srednja vrednost Naranjo skora iznosila 6,6.

Ključne reči: erythropoietin, erythropoietin side effects, anti-erythropoietin antibodies, pure red cell aplasia.

5. Eschbach JW, Kelly MR, Haley NR, Abels RI, Adamson JW. Treatment of the anemia of progressive renal failure with recombinant human erythropoietin. N Engl J Med. 1989; 321(3): 158-63.

6. Jejlkmann W. Molecular biology od erythropoietin. Intern Med. 2004; 43(8): 649-59.

7. Rang HP, Dale MM, Ritter JM, Moore PK. Farmakologija. Beograd: Data status; 2005.

8. Levin A. Recombinant of patinents and risk factors in chronic kidney disease evaluating risk factors and therapeutic strategies. Nephrol Dial Transplant 2001; 16 (Suppl 7): 57-60 .

9. Stanulović M. Biotechnological drugs with expired patent protection. In: Prostran M, Stanulović M, Marisavljević D, 
Djurić D. Farmaceutska medicina. Beograd: Medicinski fakultet Univerziteta u Beogradu, 2009: p 205.

10. Yoshimi M, Kadowaki Y, Kikuchi Y, Takahashi T. Coombs-negative autoimmune hemolytic anemia followed by anti-erythropoetin receptor antibody-associated pure red cell aplasia: a case report and review of literature. Intern Med. 2016; 55(5): 511-4.

11. Manent L, Vaglio A. Pure red cell aplasia followed by disseminated intravascular coagulation in a haemodialysis patient receiving erythropoietin-â. Nephrol Dial Transplant. 2007: 22 (5): 1465-7.

12. Okoshi Y, Imagawa S, Higuchi M, Yoshida C, Shimizu $\mathrm{S}$, Mukai $\mathrm{HY}$ et al. A patient with acquired pure red cell aplasia showing a positive antiglobulin test and the presence of inhibitor against erythroid precursors. Intern Med. 2002; 41(7): 589-92.

13. Snanoudj R, Beaudreuil S, Arzouk N, Jacq D, Casadevall N, Charpentier B et al. Recovery from pure red cell aplasia caused by anti erythropoietin antibodies after kidney transplantation. Am J transplant. 2004; 4(2):2 74-7.

14 Lee H, Yang J, Kim H, Kwon JW, Oh KH, Joo KW et al. Improvement in erythropoieis-stimulating agent-induced pure red-cell aplasia by introduction of darbepoetin- $\alpha$ when the anti-erythropoietin antibody titer declines spontaneously. J Korean Med Sci. 2010; 25(11): 1676-9.

15. Asari A, Gokal R.. Pure red cell aplasia secondary to epoetin alpha responding to darbepoetin alpha in a patient on peritoneal dialysis. J Am Soc Nephrol. 2004; 15(8): 2204-7.

16. Weber G, Gross J, Kromminga A, Loew HH, Eckardt KU. Allergic skin and systemic reactions in a patient with pure red cell aplasia and anti erythropoietin antibodies challenged with different epoetins. J Am Soc Nephrol 2002; 13(9): 2381-3.

17. Chng WJ, Tan LK, Liu TC. Cyclosporine treatment for patients with CRF who developed pure red blood cell aplasia following EPO therapy. Am J Kidney Dis. 2003. 41(3): 692-5.

\section{Correspondence to / Autor za korespondenciju}

Filip Mihajlovic

Novice Rankovic 7

34113 Stanovo - Kragujevac, Serbia

email: f.mihajlovic@yahoo.com 